\title{
EDUCAÇÃO EM SAÚDE COM OS CUIDADORES, CRIANÇAS E ADOLESCENTES DE UM AMBULATÓRIO DE SAÚDE FAMILIAR E COMUNITÁRIA
}

\author{
Bruna Cavalheiro Engel ${ }^{1}$ \\ Caroline da Silva ${ }^{2}$ \\ Chaiane dos Santos ${ }^{3}$ \\ Claiza Barretta ${ }^{4}$ \\ Fabíola Hermes Chesani ${ }^{5}$ \\ Francielly Alexandre Fabris ${ }^{6}$ \\ Marcia Aparecida Miranda de Oliveira ${ }^{7}$ \\ Maria Eduarda Luz ${ }^{8}$ \\ Mariana Francisco da Silva ${ }^{9}$ \\ Thalia Nathalia dos Santos ${ }^{10}$ \\ Luana Bertamoni Wachholz ${ }^{11}$
}

Resumo: O presente artigo é um relato de experiência do Projeto de Extensão "Humanizar e Educar em Saúde". O projeto em questão é composto por uma equipe interdisciplinar de docentes e discentes (Fisioterapia, Nutrição e Psicologia), o qual possui como objetivo principal promover a humanização em saúde junto aos cuidadores, adolescentes e crianças na sala de espera do Ambulatório de Saúde Familiar e Comunitária, situado na Universidade do Vale do Itajaí, por meio de diálogos onde estes possam relatar suas vivências, realizando trocas de saberes, através de uma conversa horizontal entre participantes e alunos. Durante o período de intervenção, 48 pacientes foram beneficiados e os encontros mostraram-se efetivos ao considerar a participação dos pacientes e seus cuidadores. As vivências apresentadas demonstram a importância da educação em saúde e da humanização na sala de espera, pois além de propiciar aos participantes novos conhecimentos, estes tornam-se integrante do processo de educação em saúde.

Palavras-chave: Humanização; Educação em saúde; Crianças; Adolescentes.

\footnotetext{
1 Psicologia/Universidade do Vale do Itajaí, Brasil. brunaengel@edu.univali.br.

2 Fisioterapia/Universidade do Vale do Itajaí, Brasil. caroline.silva3006@gmail.com.

3 Psicologia/Universidade do Vale do Itajaí. Brasil. chaiane_s@hotmail.com.

${ }^{4}$ Nutrição/universidade do Vale do Itajaí, Brasil. claizabarretta@yahoo.com.br.

${ }^{5}$ Fisioterapia/Universidade do Vale do Itajaí, Brasil. fabiola.chesani@univali.br.

${ }^{6}$ Nutrição/Universidade do Vale do Itajaí. Brasil. franfabris2010@hotmail.com.

${ }^{7}$ Psicologia/Universidade do Vale do Itajaí, Brasil. marciaoliveira3@terra.com.br.

${ }^{8}$ Nutrição/Universidade do Vale do Itajaí. Brasil. mariaeduardaluzz@gmail.com.

9 Psicologia/Universidade do Vale do Itajaí, Brasil. psicomarianafs@gmail.com.

${ }^{10}$ Fisioterapia/Universidade do Vale do Itajaí, Brasil. thalia-bc@hotmail.com.

${ }^{11}$ Nutrição/Universidade do Vale do Itajaí. Brasil. Iuana.bertamoni@hotmail.com.
} 